\title{
Semiconductor-Superlattice Parametric Oscillator as a Subterahertz and Possible Terahertz Radiation Source
}

\author{
Karl F. Renk, Andreas Rogl, Benjamin I. Stahl, Matthias Muthmann, Helmut Appel, Manish Jain, \\ Anatoly Glukhovskoy, Dieter Schuh, and Werner Wegscheider
}

Institute of Applied Physics, University of Regensburg, 93040 Regensburg, Germany

Received 8 November 2006; Revised 29 January 2007; Accepted 31 January 2007

Recommended by Ralf B. Bergmann

We describe the operation of a semiconductor-superlattice parametric oscillator (SPO) at a subterahertz frequency (near $300 \mathrm{GHz}$ ). The oscillator is driven by a microwave source (frequency near $100 \mathrm{GHz}$ ). We also present an analysis indicating that operation at frequencies above $1 \mathrm{THz}$ should be possible. The SPO is based on the ability of conduction electrons in a superlattice to perform Bloch oscillations. Broadband tunability as well as the monochromacy of a driving microwave field are transferred to the SPO.

Copyright ( 2007 Karl F. Renk et al. This is an open access article distributed under the Creative Commons Attribution License, which permits unrestricted use, distribution, and reproduction in any medium, provided the original work is properly cited.

\section{INTRODUCTION}

It is of great interest with respect to applications to develop monochromatic radiation sources for the terahertz $(\mathrm{THz})$ frequency range of $1 \mathrm{THz}$ to $10 \mathrm{THz}$ or for the sub$\mathrm{THz}$ range from $1 \mathrm{THz}$ down to $0.1 \mathrm{THz}$ (by some experts also attributed to the $\mathrm{THz}$ range). For frequencies up to about $100 \mathrm{GHz}$, well-developed current-driven semiconductor sources (e.g., the Gunn diode) are available. There is a lack of current-driven sources at higher frequencies, especially in the wavelength range around $1 \mathrm{~mm}$ (frequency $300 \mathrm{GHz}$ ) and in the submillimeter wavelength range (frequencies above $300 \mathrm{GHz}$ ).

Applications of sub-THz or THz radiation concern $[1,2]$, for example, the sensing of gas molecules or radicals (such as $\mathrm{OH}$ ) in the atmosphere, the study of spectral properties of small and large molecules, of materials or of drugs, the imaging of materials, especially biological materials, and the development of communication systems. An important application may become the security sector: imaging and sensing with $\mathrm{THz}$ or sub-THz radiation. Presently, systems operating with radiation sources based on femtosecond optical lasers are tested $[1,2]$; THz radiation can also be produced by mixing of radiation at two different frequencies produced with visible or near-infrared $\mathrm{cw}$ laser radiation [3].

Regarding simplicity and costs, current-driven semiconductor sources emitting monochromatic sub- $\mathrm{THz}$ or $\mathrm{THz}$ radiation would be most desirable. An actual development towards a current-driven monochromatic $\mathrm{THz}$ radiation source is the quantum cascade laser. Quantum cascade lasers are operated at frequencies between $1.8 \mathrm{THz}$ and $4 \mathrm{THz}[4-$ 7]. In a quantum cascade laser, it is made use of a semiconductor heterostructure which is the basis of appropriate energy levels suitable for laser action. A laser is operated at a fixed frequency that is determined by a specific heterostructure. The THz-quantum cascade laser has to be cooled to temperatures near liquid nitrogen temperature.

Another approach to realize a monochromatic sub$\mathrm{THz}$, and possibly, $\mathrm{THz}$ radiation source is the superlattice parametric oscillator (SPO). First experiments have shown that an SPO, pumped with monochromatic radiation near $100 \mathrm{GHz}$, is suitable to produce monochromatic radiation near $300 \mathrm{GHz}$ and that it is tunable [8]. The SPO is operated at room temperature.

Here, we report on an SPO using new superlattice material. We demonstrate SPO action with the new material for generation of radiation near $300 \mathrm{GHz}$ and we present a theoretical study indicating that the new material should be suitable as the basic nonlinear medium of an SPO operating at frequencies above $1 \mathrm{THz}$.

\section{PRINCIPLE OF THE SUPERLATTICE PARAMETRIC OSCILLATOR}

The principle of the SPO is illustrated in Figure 1. A semiconductor superlattice is coupled to a pump field (frequency $\omega$ ) 


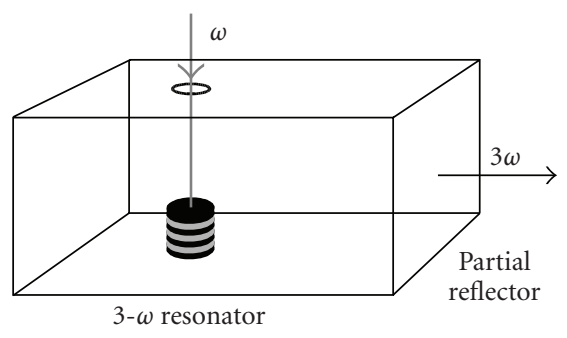

FIGURE 1: Principle of the superlattice parametric oscillator (SPO). In the SPO, pump radiation (frequency $\omega$ ) is converted to thirdharmonic radiation (frequency $3 \omega$ ). A semiconductor superlattice (with circular shape) is the active element of the SPO. The superlattice is placed in a resonator for the third-harmonic radiation. Due to the feedback delivered by the resonator, an oscillation at the thirdharmonic frequency is occurring. A partial reflector serves for output coupling of radiation from the resonator.

and to a third harmonic field (frequency $3 \omega$ ). A resonator for the 3- $\omega$ radiation delivers feedback to the superlattice. A partial reflector allows to couple third-harmonic radiation out of the resonator. The SPO is, effectively, a frequency tripler. However, due to the feedback for the third-harmonic radiation, a threshold-like onset of an oscillation occurs-the superlattice acts as an active element for the 3- $\omega$ radiation. Due to the feedback, a much higher efficiency for conversion of pump to third harmonic radiation is expected than for a conventional frequency tripler.

\section{NONLINEAR SEMICONDUCTOR-SUPERLATTICE NANOMATERIAL}

In our experiment, we have used a GaAs/AlAs superlattice (Figure 2(a)) with GaAs layers and AlAs layers in turn. Each GaAs layer consisted of 14 monolayers GaAs (monolayer thickness $0.283 \mathrm{~nm}$ ) and each AlAs layer of 2 monolayers AlAs (monolayer thickness $0.283 \mathrm{~nm}$ ). The length of a superlattice period, $a$, was thus equal to the thickness of 16 monolayers $(a=4.52 \mathrm{~nm})$. The superlattice had a length of 110 periods $(\sim 0.5 \mu \mathrm{m})$ and was homogeneously doped with silicon. The free carrier concentration was about $10^{17} \mathrm{~cm}^{-3}$.

The superlattice had been grown, by molecular beam epitaxy, on an n-GaAs layer (carrier concentration 2 . $10^{18} \mathrm{~cm}^{-3}$, thickness $600 \mathrm{~nm}$ ) that itself had been grown on a seminsulating GaAs substrate. On the top of the superlattice, there was an $\mathrm{n}$-GaAs layer (doping $2 \cdot 10^{18} \mathrm{~cm}^{-3}$, thickness $200 \mathrm{~nm}$ ). By use of photolithography and reactive ion etching, we prepared superlattice mesas. A single mesa consisted of a superlattice that was covered on top with a metal alloy (AuGeNi) which served as one of the two electric contacts. The other contact was realized via the $n-G a A s$ layer. For our experiment, we used a chip (size $150 \mu \mathrm{m} \times 150 \mu \mathrm{m}$ ) carrying about 200 superlattice mesas at distances of about $10 \mu \mathrm{m}$ from each other. Each mesa had a diameter of about $4 \mu \mathrm{m}$. Electric connection of a single mesa to a voltage source

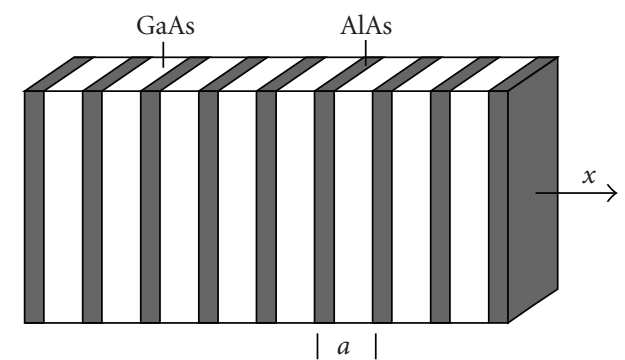

(a)

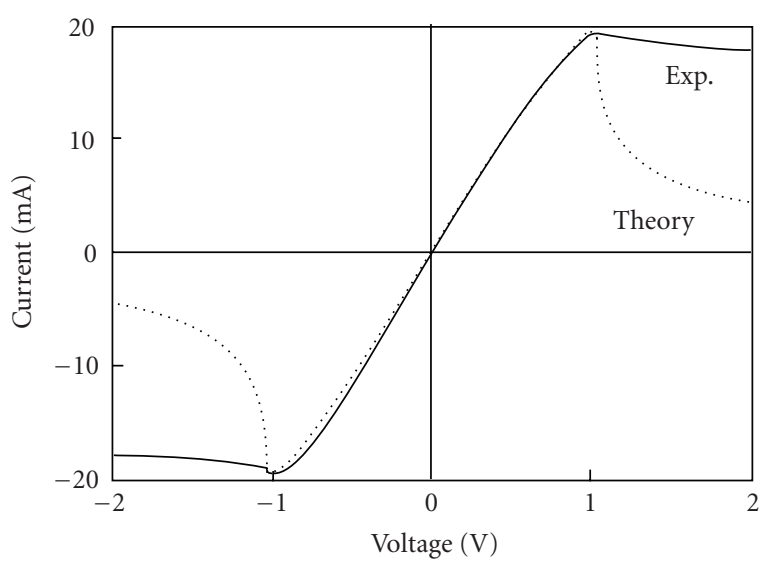

(b)

Figure 2: (a) Superlattice. The superlattice consists of GaAs and AlAs layers in turn. A GaAs layer and an adjacent AlAs layer form one superlattice period (length $a$ ) along the superlattice axis $(x)$. (b) Current-voltage curve of a superlattice mesa (diameter $4 \mu \mathrm{m}$ ). The curve indicates ohmic behavior of the superlattice around zero voltage and a nonlinear behavior above a critical voltage $(\sim 1 \mathrm{~V})$. Above the critical voltage, the current remains (at increasing voltage) almost constant (experimental curve). The theoretical curve (dotted) is obtained from the Esaki-Tsu theory, (9) to (11), taking account of a series resistance. The difference between theory and experiment can be attributed to the formation of charge density domains.

was obtained via a whisker (a thin gold wire) which we pressed on top of the mesa. The other connection was obtained via the $n$-GaAs layer and a large-area superlattice mesa.

The current-voltage curve (Figure 2(b)) was almost antisymmetric according to the symmetry of the superlattice. The curve was strongly nonlinear at voltages higher than a critical voltage $(1 \mathrm{~V})$. At voltages above the critical voltage, the current decreased slightly with increasing voltage. This indicates a negative differential resistance of the superlattice. A state of negative differential resistance corresponds to an active state of the superlattice. The ohmic resistance around zero voltage was partly due to the ohmic resistance $R_{0}$ of the superlattice and partly due to a series resistance $R_{s}$. The analysis of the current-voltage curve delivered $R_{0} \approx 10 \Omega$ and $R_{s} \approx 40 \Omega$. The series resistance was mainly due to a contact resistance of the contact between the metal alloy layer and the $\mathrm{n}$-GaAs layer on top of the superlattice. 


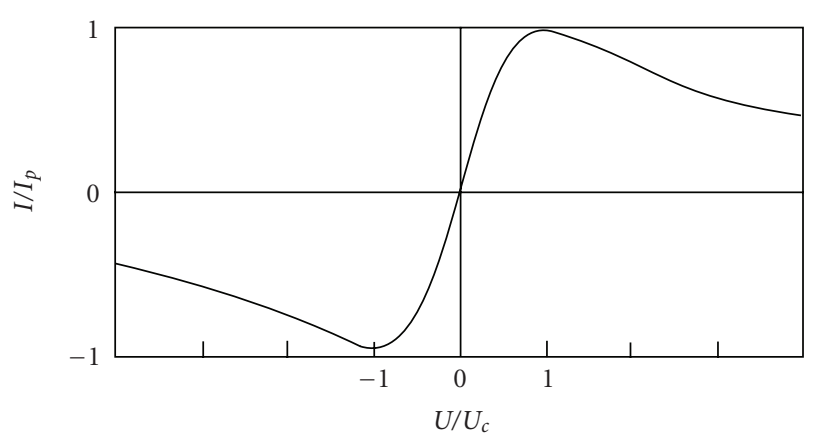

(a)
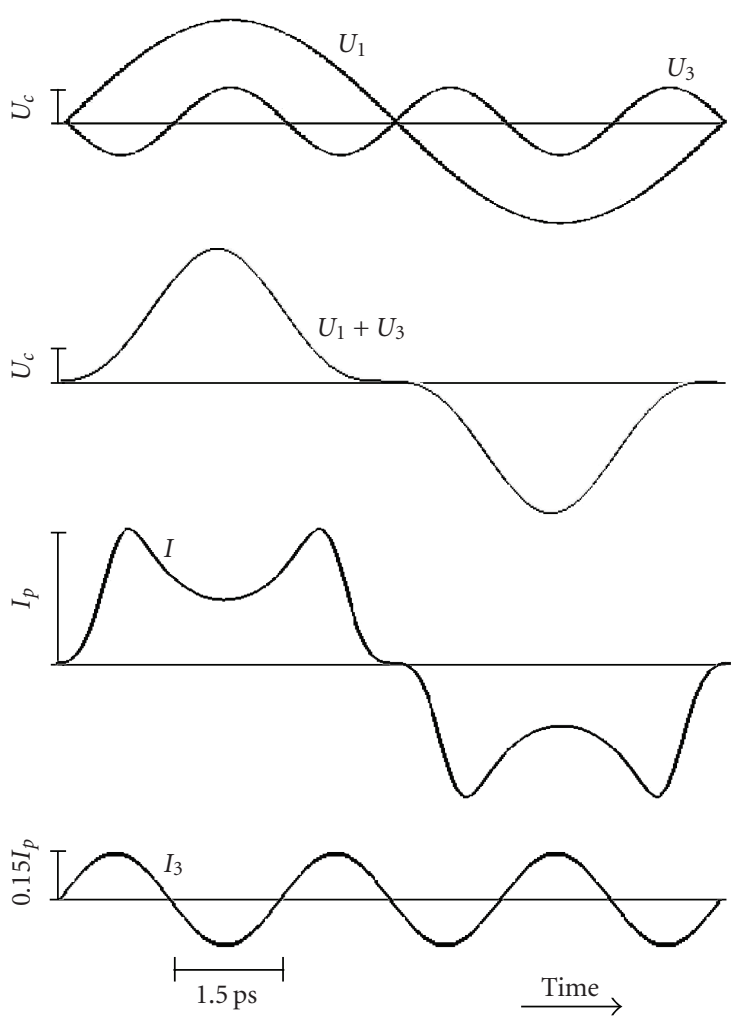

(b)

Figure 3: (a) Esaki-Tsu current-voltage curve ( $U$ = voltage; $U_{c}=$ critical voltage; $I$ = current; $I_{p}=$ peak current). (b) Mechanism of SPO action. $U_{1}$ is the pump voltage and $U_{3}$ the third-harmonic voltage across the superlattice. The total voltage, $U_{1}+U_{3}$, produces (according to the Esaki-Tsu current-voltage curve) a total current $I$ through the superlattice. The total current contains a thirdharmonic component $I_{3}$ which is the source of the third-harmonic field. $U_{3}$ and $I_{3}$ have opposite phase relative to each other, indicating gain.

We now illustrate the mechanism of the SPO action. We describe the current-voltage $(I-U)$ characteristic by an idealized curve (Figure 3(a)). The curve (Esaki-Tsu curve [8]) shows a peak current $I_{p}$ at a critical voltage $U_{c}$ and a negative differential conductance above $U_{c}$. The SPO mechanism is indicated in Figure 3(b). A high-frequency pump voltage $U_{1}$ transfers the superlattice, twice per period, into an active su-

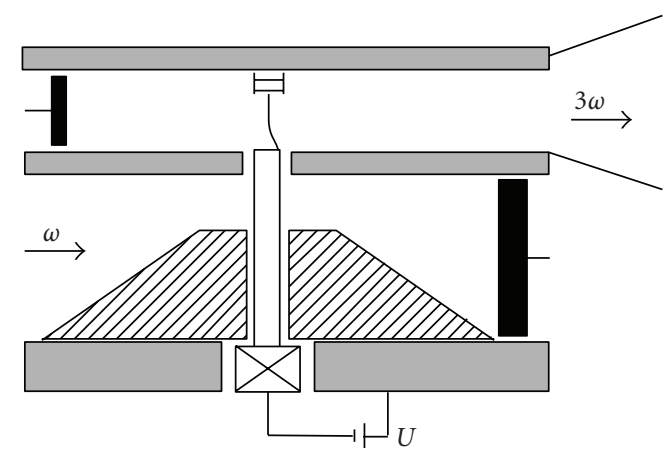

FIGURE 4: Schematic drawing of the superlattice parametric oscillator (SPO). The SPO consists of two rectangular waveguides, one for the pump radiation (frequency $\omega$ ) and the other for the thirdharmonic radiation (frequency $3 \omega$ ). They are arranged in a cross waveguide technique. The third harmonic waveguide is a resonator, with a partial reflector being formed by a mismatch between the output port of the waveguide and the horn antenna. A fin line (hatched), a stamp, and a wire act as antenna for the pump radiation. The wire acts, additionally, as antenna for the third-harmonic radiation. A static voltage $(U)$ source is used to measure the currentvoltage curve of the superlattice; SPO action is obtained for $U=0$. The pump waveguide is electromagnetically closed towards the bias circuit by use of a coaxial filter (marked by a cross).

perlattice state. An additionally applied third-harmonic voltage $U_{3}$ (as realized by the feedback with the third-harmonic resonator) results in an energy transfer from the pump field to the third-harmonic field. This is seen by analyzing the current, $I$, produced at the total voltage $U_{1}+U_{3}$, which contains a third-harmonic component $I_{3}$ with opposite phase in comparison to $U_{3}$. The time average $\left\langle U_{3} I_{3}\right\rangle$ is negative, indicating gain. The high-frequency resistance $R_{3}=U_{3} / I_{3}$ is also negative: the superlattice represents a negative resistance for the third-harmonic voltage and can therefore serve as an active element in a resonator.

\section{THE SUPERLATTICE PARAMETRIC OSCILLATOR}

Our SPO (Figure 4) consisted of a pump waveguide and a third-harmonic resonator. A chip carrying a large number of superlattice mesas was glued on one wall of the thirdharmonic resonator. A whisker antenna (a metal wire of $20 \mu$ m diameter) was pressed on one of the superlattice mesas and delivered electromagnetic coupling between the superlattice mesa and the third-harmonic resonator. A fin line (hatched) and a stamp together with the whisker served for electromagnetic coupling of the superlattice to the pump waveguide. The connection between the pump waveguide and the third-harmonic resonator was designed as a quarterwavelength coaxial line for the third harmonic to minimize third-harmonic radiation loss to the pump waveguide. By use of a coaxial filter, blocking pump radiation, a static voltage could be applied to the superlattice. Backshorts in the pump waveguide and in the third-harmonic waveguide allowed to optimize the pump power reaching the superlattice and the third-harmonic power, respectively. Radiation 
was coupled out from the third-harmonic resonator through a horn antenna. A mismatch between the output port of the third-harmonic resonator and the horn acted as partial reflector. The pump waveguide was designed for the $W$ band (cutoff frequency $60 \mathrm{GHz}$ ) and had a width of $2.54 \mathrm{~mm}$ and a height of $1.27 \mathrm{~mm}$. The third-harmonic waveguide $(1.09 \mathrm{~mm} \times 0.54 \mathrm{~mm})$ had a cutoff frequency of $131 \mathrm{GHz}$. The outer dimensions of the waveguide block were $2 \mathrm{~cm} \times 5 \mathrm{~cm} \times$ $5 \mathrm{~cm}$ (height $\times$ width $\times$ length). Attached to the block were a horn and three micrometer screws (not shown in Figure 4). One of the screws was used to press the whisker onto a superlattice mesa and the two others for shifting the backshorts.

For pumping the SPO, we used radiation of a synthesizer, amplified with a microwave amplifier (tuning range 90$94 \mathrm{GHz}$, maximum power $\sim 100 \mathrm{~mW}$ ). Third-harmonic radiation was monitored with a thermal detector (Golay cell).

\section{EXPERIMENTAL RESULTS}

The third-harmonic signal (Figure 5) showed a jump at a threshold pump power $\left(P_{\text {th }}\right)$, reached a peak immediately at $P_{\text {th }}$ and then decreased. At small pump power $P\left(P<P_{\text {th }}\right)$ the signal was due to third-harmonic generation according to the nonlinearity of the Esaki-Tsu current-voltage curve for pump field amplitudes not exceeding the critical voltage. We attribute the threshold to the onset of parametric oscillation.

The SPO signal changed with the third-harmonic resonator length (Figure 6) according to the wavelength of the radiation within the resonator. In the frequency range attainable by our pump source, corresponding to thirdharmonic frequencies from $270 \mathrm{GHz}$ to $282 \mathrm{GHz}$, thirdharmonic power varied only slightly with frequency if the backshort of the pump waveguide and the backshort of the third-harmonic resonator were appropriately adjusted.

We estimated from our results that the power of the third-harmonic radiation was of the order of $10 \mu \mathrm{W}$ corresponding to an efficiency for the conversion of pump to third-harmonic radiation of about $10^{-3}$. This value is smaller than a theoretical value $(\sim 0.1)$ and also smaller than an experimental value in a different arrangement [9]. Our present SPO device is not yet optimized with respect to the highfrequency properties. However, our results demonstrate that the new superlattice material used in the experiment is well suitable as active material in an SPO.

\section{BLOCH OSCILLATIONS AND MINIBAND TRANSPORT}

In the superlattice (Figure 2(a)), the AlAs layers present barriers of the potential for electrons moving along the superlattice axis. This has the consequence that the energy, $\varepsilon$, that is connected with the electron motion along the superlattice axis is confined to a miniband. The periodic potential gives rise to dispersion which we describe by the dispersion relation (Figure $7(\mathrm{a})$ )

$$
\varepsilon=\Delta\left(\frac{1}{2}-\frac{1}{2} \cos k a\right)
$$

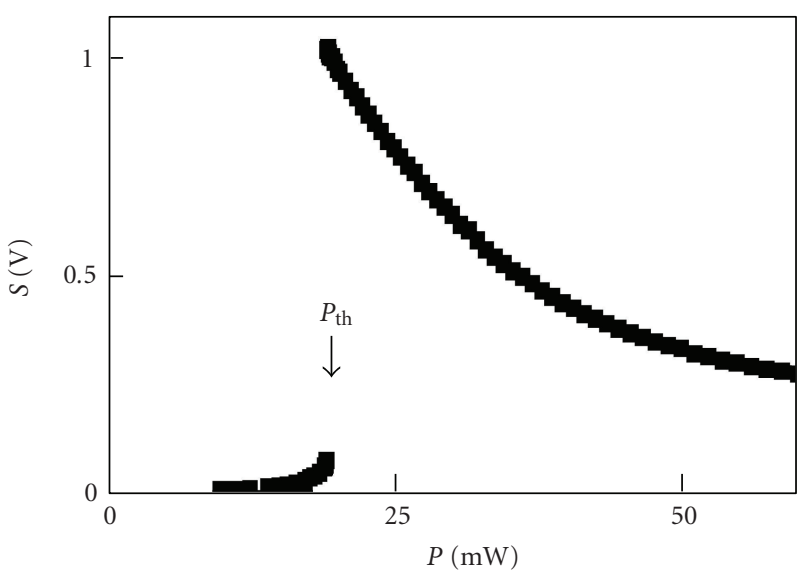

FIGURE 5: Output signal $S$ of the SPO at a third-harmonic frequency of $277.5 \mathrm{GHz}$ (pump frequency $92.5 \mathrm{GHz}$ ). The signal was obtained with a Golay cell detector. At a threshold pump power $\left(P_{\text {th }}\right)$ the signal showed, with increasing pump power $P$, a threshold-like increase. We associate the threshold with the onset of parametric oscillation.

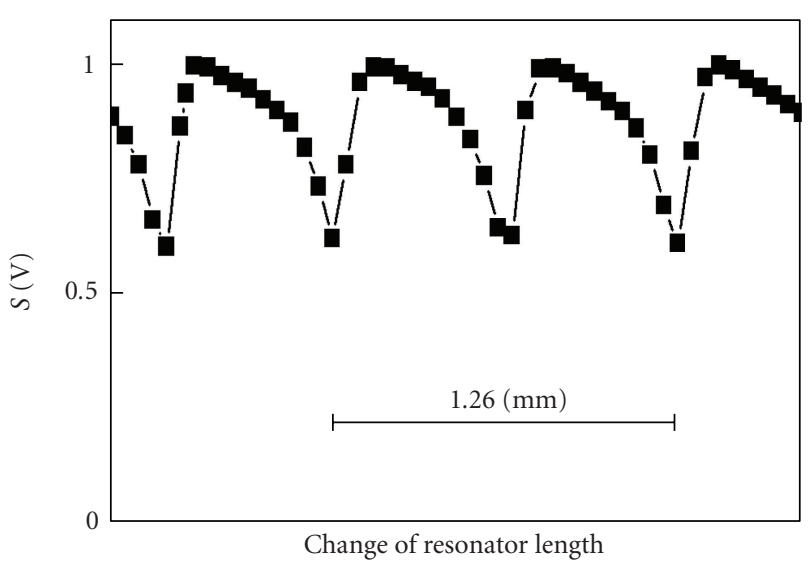

Figure 6: Signal (delivered by the Golay cell detector) at different lengths of the third-harmonic resonator (changed by shifting the corresponding backshort) at a fixed third-harmonic frequency $(277.5 \mathrm{GHz})$. The separation of the minima $(0.53 \mathrm{~mm})$ corresponded to half a wavelength of the radiation within the thirdharmonic resonator.

where $k$ is the wave vector along the superlattice axis and $\Delta$ the width of the miniband. The energy has a minimum $(\varepsilon=0)$ for $k=0$ and maxima at the mini-Brillouin zone boundaries $k= \pm \pi / a$. The miniband is separated from the next higher miniband by a minigap. An electron has the total energy $\varepsilon+\left(2 m^{*}\right)^{-1} \hbar^{2} k_{\perp}^{2}$, where $m^{*}$ is the effective mass and $k_{\perp}$ the wavevector for the propagation perpendicular to the superlattice axis; the perpendicular motion is similar to that of a conduction electron in a GaAs crystal $\left(m^{*}=0.07 m_{0} ; m_{0}=\right.$ electron mass). A calculation of the miniband width for our superlattice delivered the miniband width $\Delta \sim 140 \mathrm{meV}$ and a minigap to the next higher miniband of $\sim 180 \mathrm{meV}$. 


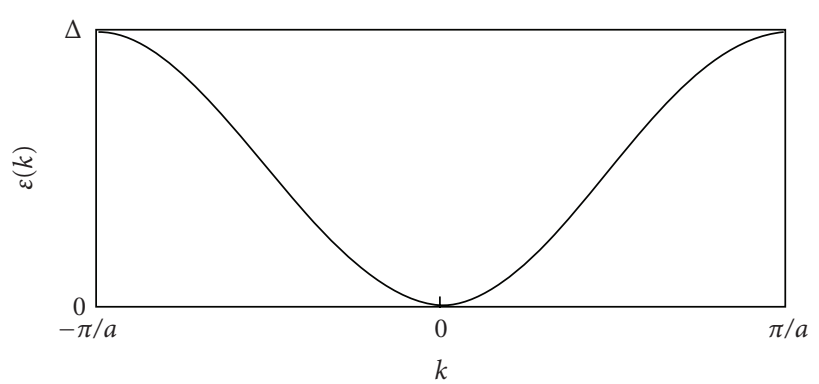

(a)

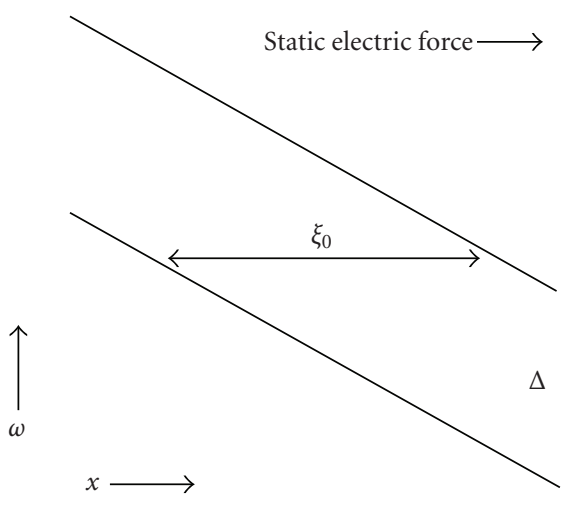

(b)

FIGURE 7: (a) Dispersion relation for a miniband electron propagating along the superlattice axis $(\varepsilon=$ energy and $k=$ wave vector of an electron propagating along the superlattice axis; $a=$ superlattice period; $\Delta=$ miniband width). (b) Trajectory (horizontal line with arrows; length $\xi_{0}$ ) for the Bloch oscillation of a miniband electron under the influence of a static electric force directed along the superlattice axis $(x)$. The lower of the two parallel lines symbolizes the bottom of the miniband $(\varepsilon=0)$ and the upper line the upper boundary $(\varepsilon=\Delta)$. The tilt of the miniband accounts for a decrease of the potential energy along the direction of the static electric force. The electron propagates from the bottom to the upper boundary of the miniband, where it undergoes a Bragg reflection and moves back to the bottom to start a new oscillation cycle. On a trajectory, the sum of potential and kinetic energy of an electron is constant.

Under the action of a static field, an electron is accelerated, reaches the mini-Brillouin zone boundary, and is then Bragg reflected and decelerated. Then, it is accelerated again. The electron performs a Bloch oscillation with the Bloch frequency

$$
\omega_{B}=\frac{e a E}{\hbar},
$$

where $e$ is the electron charge and $E$ the static-field strength ( $\hbar$, Planck's constant). This follows from the acceleration theorem (Newton's law for a crystal electron),

$$
\hbar \dot{k}=e E .
$$

The wave vector increases linearly with time $t$,

$$
k a=\omega_{B} t,
$$

and the group velocity

$$
v_{g}=\frac{1}{\hbar} \frac{\partial \varepsilon}{\partial k}=\frac{\Delta a}{2 \hbar} \sin \omega_{B} t
$$

oscillates with the Bloch frequency. By integration, the spatial position (trajectory) $\xi(t)=\int_{0}^{t} v_{g} d t$ is obtained. It follows that the electron performs a Bloch oscillation in space with the trajectory (Figure 7(b))

$$
\xi=\xi_{0}\left(\frac{1}{2}-\frac{1}{2} \cos \omega_{B} t\right),
$$

where

$$
\xi_{0}=\frac{\Delta}{\hbar \omega_{B}} a
$$

is the length of the trajectory. For $E=10 \mathrm{kV} / \mathrm{cm}$, the Bloch frequency for an electron in our superlattice is $(1 / 2 \pi) \omega_{B} \sim$ $1 \mathrm{THz}$ and the length of the trajectory $\xi_{0} \sim 30 a$. The electron oscillates over a large number of superlattice periods.

The description of the Bloch oscillation implies: under the influence of a static electric force along the superlattice axis, a conduction electron at zero energy $(\varepsilon=0)$ gains energy until it reaches the zone boundary $(\varepsilon=\Delta)$. Then, the electron undergoes Bragg reflection and moves against the electric force until it reaches again zero energy and begins a new oscillation cycle. The matter wavelength of the electron is almost infinitely large at $k=0$ and decreases with increasing energy until the wavelength is equal to twice the period of the superlattice giving rise to Bragg reflection. The Bragg reflection is an extremely nonlinear process and is the origin of the nonlinearity of transport properties of a superlattice. The actual basis of the nonlinear transport is the interplay of Bloch oscillations, that is, Bragg reflections, with intraminiband relaxation.

A Bloch oscillation is interrupted by relaxation. The electron looses energy by emission of a phonon and starts a Bloch oscillation with a new phase at a lower energy. This leads to a drift in the direction of the static force. The average drift velocity is

$$
v=\frac{1}{\tau} \int_{0}^{\infty} \exp \left(\frac{-t}{\tau}\right) v_{g}(t) d t
$$

where $\tau$ is the intraminiband relaxation time. By integration, the Esaki-Tsu characteristic [8] is obtained:

$$
v=v_{p} \frac{2 E / E_{c}}{1+E^{2} / E_{c}^{2}}=v_{p} \frac{2 \omega_{B} \tau}{1+\omega_{B}^{2} \tau^{2}},
$$

where

$$
v_{p}=\frac{\Delta a}{4 \hbar}
$$

is the peak-drift velocity occurring at a critical field $E_{c}=$ $\hbar(e a \tau)^{-1}$.

The current, $I$, through the superlattice is given by

$$
I=N e A v,
$$


where $N$ is the carrier concentration and $A$ the area of the superlattice. Equation (9) delivers, with $U=E / L$ and $U_{c}=$ $E_{c} / L$, where $L$ is the length of the superlattice, the Esaki-Tsu current-voltage curve (Figure 3(a)). The superlattice has an ohmic resistance $R_{0}=(1 / 2) U_{c} / I_{p}$ around zero voltage and shows a negative differential resistance for voltages larger than $U_{c}$. We obtained the theoretical current-voltage curve (dotted in Figure 2(b)) that is in accordance with the experimental curve for voltages up to the critical voltage, taking into account a series resistance. The voltage of maximum current corresponded to a critical field $E_{c} \approx 10 \mathrm{kV} / \mathrm{cm}$, delivering a relaxation time $(\sim 150 \mathrm{fs})$ that is characteristic for miniband transport in GaAs/AlAs superlattices. The relaxation was mainly due to emission of polar optic phonons. The peak current density $\left(\sim 160 \mathrm{kA} / \mathrm{cm}^{2}\right)$ was in agreement with the value calculated with the Esaki-Tsu formula, taking account of a small correction due to the finite temperature $(300 \mathrm{~K})$ of the superlattice. The difference between the theoretical and the experimental curves for $U>U_{c}$ was due to the occurrence of space charge domains in the range of negative differential conductance. In the following treatment of SPO action, we neglect the possibility of the occurrence of domains.

Bloch oscillations have been observed by femtosecond optical techniques $[10,11]$. Transport studies have shown that miniband transport occurs [12]. It has been made use of the negative differential conductance for the development of superlattice oscillators driven by a static field; up to now, oscillation frequencies up to almost $200 \mathrm{GHz}$ have been reached [13-19]. The nonlineartiy can be used for frequency multiplication [20-22], for heterodyne detection [23] and for direct detection of ultrashort sub- $\mathrm{THz}$ and $\mathrm{THz}$ radiation pulses, up to frequencies above $10 \mathrm{THz}$ [24-26].

\section{THEORY OF SPO ACTION}

For SPO action, a pump field (amplitude $\widehat{E}_{1}$ )

$$
E_{1}=\widehat{E}_{1} \cos \omega t
$$

and its third-harmonic field (amplitude $\widehat{E}_{3}$ )

$$
E_{3}=\widehat{E}_{3} \cos 3 \omega t
$$

are superimposed in a superlattice. The total field

$$
E(t)=\widehat{E}_{1} \cos \omega t+\widehat{E}_{3} \cos 3 \omega t
$$

causes a time dependent drift velocity $v(t)$. This contains a third-harmonic drift-velocity component

$$
v_{3}=\hat{v}_{3} \cos 3 \omega t
$$

which is the source of a third-harmonic current, where $\hat{v}_{3}$ is the amplitude of the third-harmonic drift velocity component.

The Esaki-Tsu formula, (9), describes the electron drift velocity for a superlattice in a static field. The formula can also be used for describing SPO action, that is, to determine $v(t)$ and $\hat{v}_{3}$, if the frequency of the third-harmonic field is small compared to the electron relaxation frequency, $3 \omega \tau \ll 1$. This quasistationary case is illustrated in Figure 3. For the SPO action at $300 \mathrm{GHz}$, we have $3 \omega \tau \approx 0.3$. For this frequency, the quasistatic theory is at its limits.

In the general case that $\omega \tau$ is not small compared to 1 , we cannot apply the Esaki-Tsu characteristic for the description of the electron motion. Instead, we derive the time dependent drift velocity for a miniband electron in a time dependent electric field $E(t)$ directly from the acceleration theorem, (3). It follows that

$$
k\left(t, t_{0}\right)=\frac{e}{\hbar} \int_{t_{0}}^{t} E\left(t^{\prime}\right) d t^{\prime},
$$

where $t_{0}$ is the time at which the acceleration of an electron starts, at a field $E\left(t_{0}\right)$. If the electron moves unscattered until the time $t$, its group velocity is

$$
v_{g}\left(t, t_{0}\right)=\frac{\Delta a}{2 \hbar} \sin \left[\int_{t_{0}}^{t} \omega_{B}\left(t^{\prime}\right) d t^{\prime}\right]
$$

where

$$
\omega_{B}(t)=\frac{1}{\hbar} e a E(t)=\frac{1}{\hbar} e a\left(\hat{E}_{1} \cos \omega t+\hat{E}_{3} \cos 3 \omega t\right)
$$

is the instantaneous Bloch frequency. We can interprete the instantaneous Bloch frequency as an instantaneous Bragg scattering frequency. The maximum instantaneous Bloch (Bragg scattering) frequency is obtained twice per period of the pump field and has the value

$$
\omega_{B, \max }=\frac{1}{\hbar} e a\left(\widehat{E}_{1}+\widehat{E}_{3}\right) .
$$

The maximum Bloch frequency corresponds to a minimum length of an instantaneous trajectory

$$
\xi_{0, \min }=\frac{\Delta}{\hbar \omega_{B, \max }} a .
$$

The time-dependent drift velocity is obtained by averaging the group velocities over all starting times,

$$
\begin{aligned}
v(t) & =\int_{-\infty}^{t} p\left(t, t_{0}\right) v_{g}\left(t, t_{0}\right) d t_{0} \\
& =2 v_{p} \int_{-\infty}^{t}\left\{p\left(t, t_{0}\right) \sin \left[\int_{t_{0}}^{t} \omega_{B}\left(t^{\prime}\right) d t^{\prime}\right]\right\} d t_{0},
\end{aligned}
$$

where

$$
p\left(t, t_{0}\right)=\frac{1}{\tau} \exp \left[-\left(t-t_{0}\right) \tau^{-1}\right]
$$

is the probability that an electron did not undergo a relaxation process in the time interval between $t_{0}$ and $t$. A derivation of $v(t)$ by use of a Boltzmann equation [27] leads to the same result. If a finite temperature $T$ is taken into account, $v_{p}$ has to be replaced by $v_{p} I_{1}(z) / I_{0}(z)$ where $I_{0}$ and $I_{1}$ are the Bessel functions of the zeroth and first order and $z=\Delta\left(2 k_{B} T\right)^{-1}$; for $k_{B} T \ll \Delta$, we have $I_{1} / I_{0}=1\left(k_{B}\right.$, Boltzmann constant). For our superlattice $(\Delta \sim 140 \mathrm{meV})$ at room 


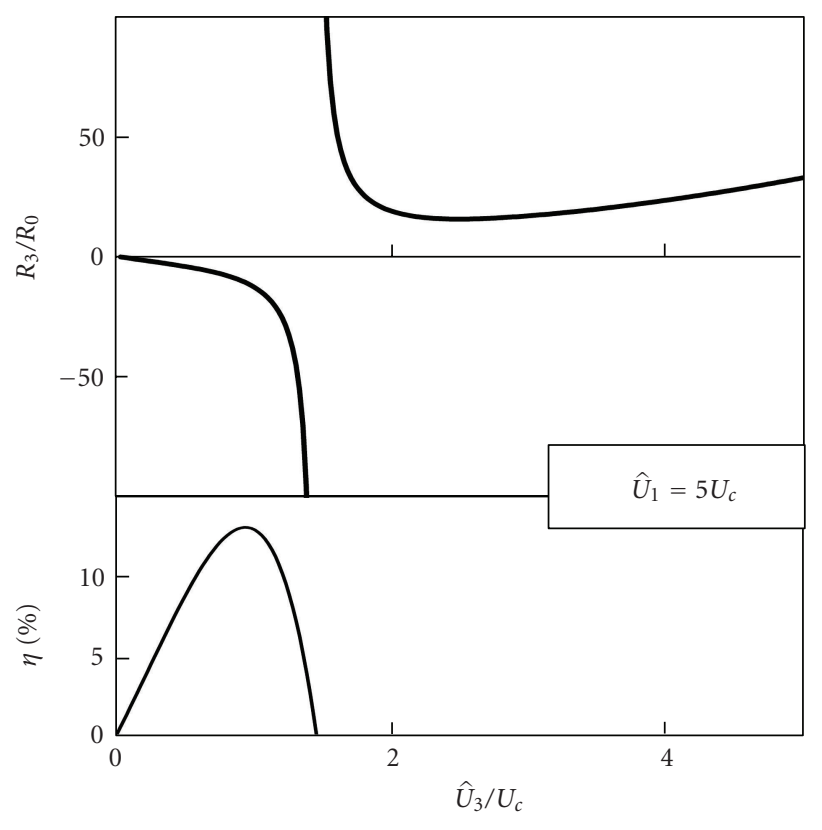

Figure 8: Calculated third-harmonic resistance $R_{3}$ and efficiency $\eta$ for a $300-\mathrm{GHz}$ SPO, at a fixed amplitude $\hat{U}_{1}$ of the pump voltage $\left(R_{0}=\right.$ Ohmic resistance of the superlattice and $U_{c}=$ critical voltage). The third-harmonic resistance $R_{3}$ is negative for a large range of the third-harmonic amplitude $\hat{U}_{3}$ (between 0 and $\left.1.6 U_{c}\right)$ and becomes positive for very large values of $\hat{U}_{3}\left(>1.6 U_{c}\right)$. A negative value of $R_{3}$ indicates gain for the third harmonic. In the case of gain, energy is transferred from the pump field to the third-harmonic field. With increasing amplitude $\hat{U}_{3}$ the efficiency increases, up to an optimum and then decreases to zero (for $\hat{U}_{3} \approx$ $\left.1.6 U_{c}\right)$.

temperature $(T=300 \mathrm{~K})$ the ratio was slightly smaller than $1\left(I_{1} / I_{0} \sim 0.8\right)$.

We calculated $v(t)$ and (by Fourier analysis) $\hat{v}_{3}$ for different values of the amplitudes $\hat{E}_{1}$ and $\hat{E}_{3}$ and determined the amplitude of the third harmonic current from the relation $\hat{I}_{3} / I_{p}=\hat{v}_{3} / v_{p}$ and the amplitude of the third-harmonic voltage from $\hat{U}_{3} / U_{c}=\widehat{E}_{3} / E_{c}$. From the amplitudes, we obtained the resistance $R_{3}=\hat{U}_{3} / \hat{I}_{3}=2\left(\hat{U}_{3} / U_{p}\right)\left(I_{c} / \hat{I}_{3}\right) R_{0}$. We also calculated the amplitude of the drift velocity, $\hat{v}_{1}$, at the pump frequency and the current amplitude $\hat{I}_{1} / I_{p}=\hat{v}_{1} / v_{p}$ and the voltage amplitude $\hat{U}_{1} / U_{c}=\widehat{E}_{1} / E_{c}$. From the power $P_{1}=(1 / 2) \hat{U}_{1} \widehat{I}_{1}$ and the power $P_{3}=(1 / 2) \hat{U}_{3} \hat{I}_{3}$, we obtained the efficiency $\eta=P_{3} / P_{1}$ for the conversion of pump to the third-harmonic radiation.

A theoretical result for an oscillation frequency which corresponds to our experiment is shown in Figure 8. We have chosen a pump voltage $\hat{U}_{1}=3 U_{c}$. The third-harmonic resistance $R_{3}$ is negative at small amplitude $\hat{U}_{3}$, its absolute value increases with $\hat{U}_{3}$. This indicates the possibility of a self oscillation of the SPO, with feedback delivered by the resonator. The efficiency has an optimum of about 10 percent. For too large amplitudes $\widehat{U}_{3}, \eta$ decreases and finally becomes zero.

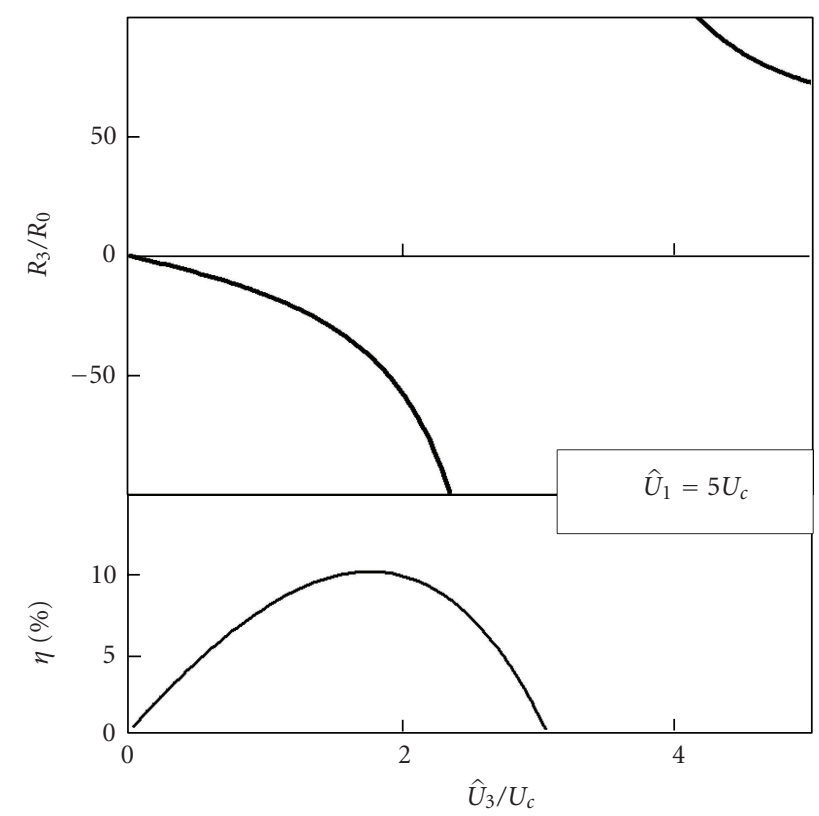

FIGURE 9: Calculated-third harmonic resistance $R_{3}$ and efficiency $\eta$ for a $3 \mathrm{THz} \mathrm{SPO}$ at a fixed amplitude $\hat{U}_{1}$ of the pump voltage $\left(R_{0}=\right.$ Ohmic resistance of the superlattice and $U_{c}=$ critical voltage). The resistance for the third harmonic, $R_{3}$, is negative for $\hat{U}_{3} / U_{c}<2.3$ indicating gain for the third harmonic. The efficiency has a maximum for $\hat{U}_{3} \approx 2 U_{c}$.

Another theoretical result (Figure 9) indicates the possibility to operate an SPO at $3 \mathrm{THz}$. An efficiency of 10 percent may be, in principle, achievable at a pump voltage $\hat{U}_{1}=5 U_{c}$. A comparison of our results shows that the condition to reach an efficiency of about 10 percent is different for the 300-GHz and the 3-THz SPO. For the higher frequency, larger voltage amplitudes are necessary. These are joint with lower current amplitudes. By appropriate impedance matching of pump and resonance circuit, the necessary coupling of pump and third-harmonic radiation should be achievable. As a main result we find, theoretically, that pumping with, for example, a $10 \mathrm{~mW}$ source delivers at $300 \mathrm{GHz}$ and $3 \mathrm{THz}$ almost the same third-harmonic power of about $1 \mathrm{~mW}$. By use of another design of the superlattice, it should in principle be possible to increase both pump and third-harmonic power. The reason for almost the same efficiency for the quasistatic and dynamic case $(3 \omega \tau \approx 0.3$ and $3 \omega \tau \approx 3$, resp. $)$ is the strong nonlinearity caused by Bragg reflections of the miniband electrons. Further and more detailed theoretical investigations are published elsewhere $[28,29]$; in these studies, it is also shown that space charge domains can be absent.

\section{THE UPPER LIMIT FREQUENCY FOR SPO ACTION}

A superlattice is suitable as active medium in an SPO under the condition that instantaneous Bragg reflection processes are possible at the field amplitudes necessary for operating 
an SPO, that is, if

$$
\xi_{0, \min } \gg a \text {. }
$$

If $\xi_{0, \min } \leq a$, the electron is localized to one superlattice spatial period.

It follows that the upper limit frequency for SPO action is given by the condition

$$
\hbar \omega_{B, \max } \ll \Delta .
$$

For our superlattice, we find for a third-harmonic frequency of $3 \mathrm{THz}$ (with the necessary field amplitudes $\hat{U}_{1}=5 U_{c}$ and $\left.\hat{U}_{3} \sim U_{c}\right)$ that $\xi_{0, \text { min }} \approx 5 a$. Thus, the condition $\xi_{0, \text { min }} \gg a$ is fulfilled. The frequency of $3 \mathrm{THz}$ represents an upper limit for SPO action of our superlattice.

We estimate for a superlattice used in the first SPO experiment [8], with $\Delta \sim 24 \mathrm{meV}$ (18 monolayers GaAs and 4 monolayers AlAs, per superlattice period), a limit frequency of about $0.6 \mathrm{THz}$.

\section{DISCUSSION}

We have described an SPO with superlattice material that should be suitable to realize a THz SPO. Starting with a 100$\mathrm{GHz}$ or a $200-\mathrm{GHz}$ microwave pump source it should, in principle, be possible to use SPOs in series to produce radiation up to few $\mathrm{THz}$.

However, there are several technical problems to be solved: an SPO of a higher power requires the improvement of the arrangement. It is necessary to develop submillimetersize waveguides, filters, and antennas of high quality, and it is necessary to appropriately couple a superlattice to the waveguide structure. In our present experiment, we have used a mesa shaped superlattice. This allowed us to use different superlattice geometries (especially of different diameters) in a simple way. However, a stable oscillator requires the use of a quasiplanar superlattice device (as it has been used for the first SPO experiment [9]). Furthermore, more efficient cooling of the superlattice should be introduced allowing for larger area and higher pump power and reducing thermal heating.

\section{ACKNOWLEDGMENT}

The project has been supported by the Deutsche Forschungsgemeinschaft through the Graduiertenkolleg 638: "nonlinearity and nonequilibrium in condensed matter."

\section{REFERENCES}

[1] D. Mittleman, Ed., Sensing with Terahertz Radiation, Springer, Berlin, Germany, 2003.

[2] R. E. Miles, P. Harrison, and D. Lippens, Eds., Terahertz Sources and Systems, Kluwer, London, UK, 2001.

[3] K. J. Siebert, H. Quast, R. Leonhardt, et al., "Continuous-wave all-optoelectronic terahertz imaging," Applied Physics Letters, vol. 80, no. 16, pp. 3003-3005, 2002.

[4] R. Köhler, A. Tredicucci, F. Beltram, et al., "Terahertz semiconductor-heterostructure laser," Nature, vol. 417, no. 6885, pp. 156-159, 2002.
[5] L. Mahler, R. Köhler, A. Tredicucci, et al., "Single-mode operation of terahertz quantum cascade lasers with distributed feedback resonators," Applied Physics Letters, vol. 84, no. 26, pp. 5446-5448, 2004.

[6] B. S. Williams, S. Kumar, Q. Hu, and J. L. Reno, "Resonantphonon terahertz quantum-cascade laser operating at $2.1 \mathrm{THz}$ $(\lambda \simeq 141 \mu \mathrm{m})$," Electronics Letters, vol. 40, no. 7, pp. 431-433, 2004 .

[7] J. Alton, S. S. Dhilon, C. Sirtori, et al., "Buried waveguides in terahertz quantum cascade lasers based on two-dimensional surface plasmon modes," Applied Physics Letters, vol. 86, no. 7, Article ID 071109, 3 pages, 2005.

[8] L. Esaki and R. Tsu, "Superlattice and negative differential conductivity in semiconductors," IBM Journal of Research and Development, vol. 14, no. 1, p. 61, 1970.

[9] K. F. Renk, B. I. Stahl, A. Rogl, et al., "Subterahertz superlattice parametric oscillator," Physical Review Letters, vol. 95, no. 12, Article ID 126801, 4 pages, 2005.

[10] J. Feldmann, K. Leo, J. Shah, et al., "Optical Investigation of Bloch oscillations in a semiconductor superlattice," Physical Review B, vol. 46, no. 10, pp. 7252-7255, 1992.

[11] K. Leo, High-Field Transport in Semiconductor Superlattices, Springer, Berlin, Germany, 2003.

[12] A. Sibille, J. F. Palmier, H. Wang, and F. Mollot, "Observation of Esaki-Tsu negative differential velocity in GaAs/AlAs superlattices," Physical Review Letters, vol. 64, no. 1, pp. 52-55, 1990.

[13] K. F. Renk, A. Meier, B. I. Stahl, et al., "Operation of a Bloch oscillator," 2005, http://arxiv.org/abs/cond-mat/0502283.

[14] M. Häußler, R. Scheuerer, K. F. Renk, Yu. Kuschurinov, and D. G. Pavel'ev, "Microwave frequency multiplication by use of space charge domains in semiconductor superlattice," Electronics Letters, vol. 39, no. 7, pp. 628-629, 2003.

[15] M. Jain, H. Appel, A. Meier, W. Wegscheider, and K. F. Renk, "Semiconductor-superlattice oscillator with superlattices connected in parallel and series as active elements for generation of millimetre waves," IEE Proceedings: Microwaves, Antennas and Propagation, vol. 153, no. 5, pp. 441-446, 2006.

[16] E. Schomburg, R. Scheuerer, S. Brandl, et al., "InGaAs/InAlAs superlattice oscillator at $147 \mathrm{GHz}$," Electronics Letters, vol. 35, no. 17, pp. 1491-1492, 1999.

[17] E. Schomburg, M. Henini, J. M. Chamberlain, et al., "Selfsustained current oscillation above $100 \mathrm{GHz}$ in a GaAs/AlAs superlattice," Applied Physics Letters, vol. 74, no. 15, pp. 21792181, 1999.

[18] H. Eisele, M. Naftaly, and R. Kamoua, "Generation of submillimeter-wave radiation with GaAs tunnett Diodes and InP gunn devices in a second or higher harmonic mode," International Journal of Infrared and Millimeter Waves, vol. 26, no. 1, pp. 1-14, 2005.

[19] H. Eisele, "Active two-terminal devices as sources at $\mathrm{THz}$ frequencies: concepts, performance, and trends," in ICONO 2005: Nonlinear Laser Spectroscopy, High Precision Measurements, and Laser Biomedicine and Chemistry, vol. 6257 of Proceedings of SPIE, pp. 10 pages, St. Petersburg, Russia, May 2005.

[20] S. Winnerl, E. Schomburg, S. Brandl, et al., "Frequency doubling and tripling of terahertz radiation in a GaAs/AlAs superlattice due to frequency modulation of Bloch oscillations," Applied Physics Letters, vol. 77, no. 9, pp. 1259-1261, 2000.

[21] F. Klappenberger, K. F. Renk, P. Renk, et al., "Semiconductorsuperlattice frequency multiplier for generation of submillimeter waves," Applied Physics Letters, vol. 84, no. 19, pp. 3924-3926, 2004. 
[22] R. Scheuerer, M. Häußler, K. F. Renk, et al., "Frequency multiplication of microwave radiation by propagating space-charge domains in a semiconductor superlattice," Applied Physics Letters, vol. 82, no. 17, pp. 2826-2828, 2003.

[23] K. F. Renk, A. Rogl, B. I. Stahl, et al., "Semiconductorsuperlattice frequency mixer for detection of submillimeter waves," to appear in International Journal of Infrared and Millimeter Waves.

[24] S. Winnerl, W. Seiwerth, E. Schomburg, et al., "Ultrafast detection and autocorrelation of picosecond $\mathrm{THz}$ radiation pulses with a GaAs/AlAs superlattice," Applied Physics Letters, vol. 73, no. 20, pp. 2983-2985, 1998.

[25] F. Klappenberger, A. A. Ignatov, S. Winnerl, et al., "Broadband semiconductor superlattice detector for $\mathrm{THz}$ radiation," Applied Physics Letters, vol. 78, no. 12, pp. 1673-1675, 2001.

[26] F. Klappenberger and K. F. Renk, "Transient-pulse nonlinear spectroscopy with the radiation of a multimode THz gas laser," International Journal of Infrared and Millimeter Waves, vol. 24, no. 9, pp. 1405-1414, 2003.

[27] A. A. Ignatov, E. Schomburg, J. Grenzer, K. F. Renk, and E. P. Dodin, "THz-field induced nonlinear transport and dc voltage generation in a semiconductor superlattice due to Bloch oscillations," Zeitschrift für Physik B Condensed Matter, vol. 98, no. 2, pp. 187-195, 1995.

[28] K. F. Renk, A. Rogl, and B. I. Stahl, "Semiconductorsuperlattice parametric oscillator for generation of subterahertz and terahertz waves," Journal of Luminescence, vol. 125, pp. 252-258, 2007.

[29] B. I. Stahl, A. Rogl, and K. F. Renk, "A theoretical study of operation conditions for a terahertz superlattice parametric oscillator," Physics Letters A, vol. 359, no. 5, pp. 512-515, 2006. 

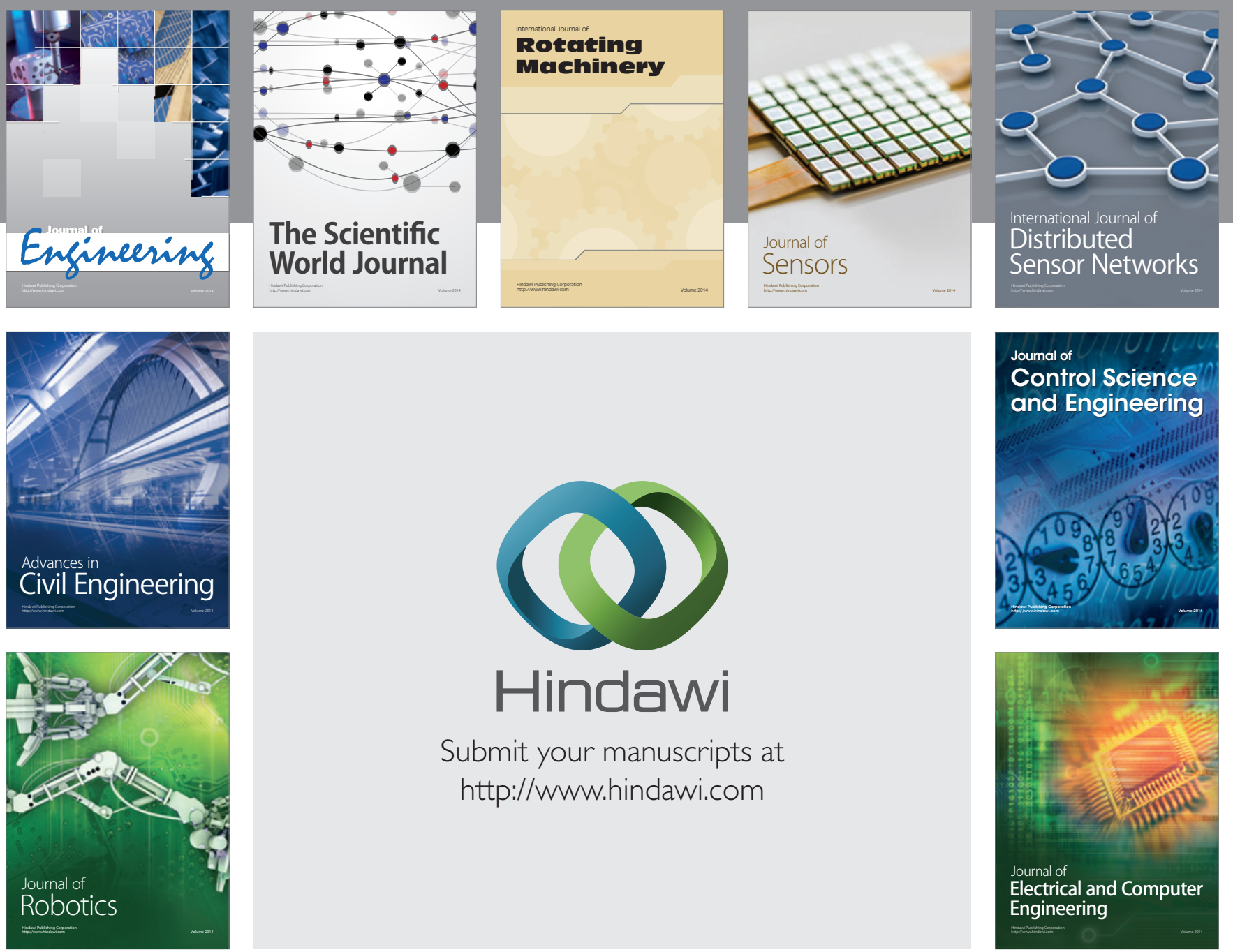

Submit your manuscripts at

http://www.hindawi.com
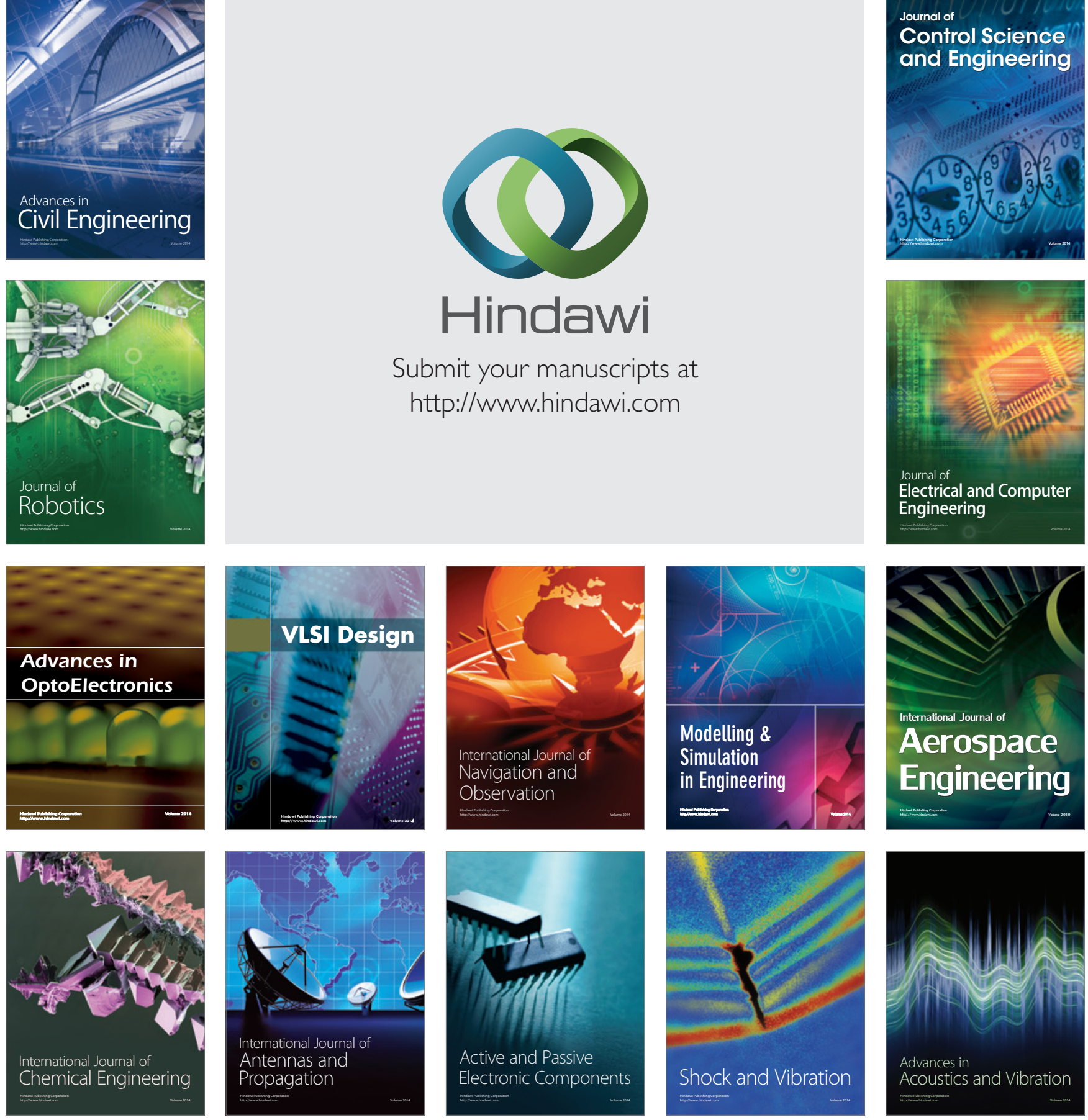